\title{
Increasing Spectral Efficiency by Managing Adjacent Channel Interference
}

\author{
Tobias Renk, Clemens Kloeck, Christian Koerner, and Friedrich K. Jondral \\ Institut für Nachrichtentechnik \\ Universität Karlsruhe (TH) \\ Email: \{renk, kloeck, koerner, fj\}@int.uni-karlsruhe.de
}

\begin{abstract}
Due to the ever-increasing demands in wireless communications, spectrum seems to be a scarce resource today. Hence, new techniques that deal with the issue of spectrum management have to be developed. In this paper, possibilities to reduce the guard band between consecutive channels dynamically are investigated, thus improving spectral efficiency at the expense of an increasing amount of adjacent channel interference. In a first consideration, the strategy of shifting carrier frequencies is investigated. A second approach is the adaptive adjustment of roll-off factors when root raised cosine filters are used for pulse shaping as it is the case in $3 \mathrm{G}$ mobile communication systems. Moreover, an algorithm for dynamic guard band adaptation is developed. A simulation environment is set up and the simulation results are presented and discussed as well.
\end{abstract}

\section{INTRODUCTION}

In wireless communications energy from a transmitting system might spill over into consecutive frequency bands causing capacity and performance degradation (see Fig. 1). This effect is known as Adjacent Channel Interference (ACI). A lot of questions arise with the term ACI. What are the reasons for ACI? On which parameters does ACI depend? And how does ACI eventually affect the performance of communication systems? ACI occurs, e.g., if several communication systems are operating within the same geographical area. Further reasons are transmit and receive filter inadequacies and power amplifier non-linearities [1], [2]. The following listing shows different parameters that influence the amount of ACI:

- Spatial distance between operating systems

- Antenna arrangement

- Propagation environment

- Required quality of transmission

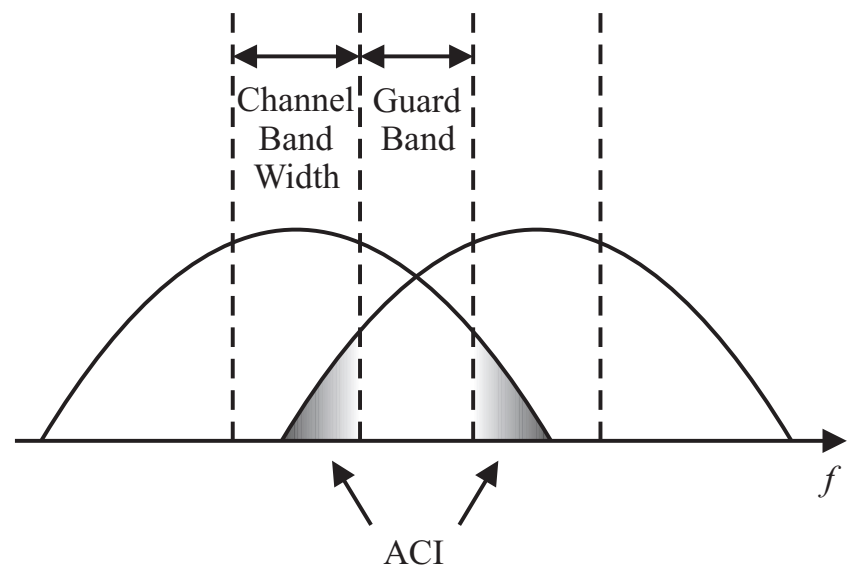

Figure 1: Adjacent channel interference

- Modulation scheme

- Transmit power

- Pulse shaping filter

- Guard band between consecutive channels

A lot of previous work has been done in the research area of ACI. Obviously, the simplest approach to reduce ACI is the use of sharper filters. However, this increases cost and complexity. Further approaches deal with suppression of ACI by applying linear and decision feedback equalizers [3], using successive cancelation or joint modulation techniques [4], [5]. In [6], two digital algorithms for suppression of ACI in FM-receivers are presented. However, these approaches increase the complexity of the receiver as well. The effects of ACI on the capacity of Frequency Division Multiple Access (FDMA) cellular systems and on the performance of differentially detected Gaussian Minimum Shift Keying (GMSK) signals have been investigated by S. Golestaneh et al. [7] and P. T. Mathiopoulos et al. [8], respectively. B. Vaghani and C. Beard used linear, higher order modulation schemes in order to reduce ACI and improve spectral efficiency [9], [10].

In this paper, the aim of increasing the overall spectral efficiency is picked up. The ideas of either shifting carrier frequencies or changing roll-off factors dynamically are investigated. For this purpose two systems are considered, 


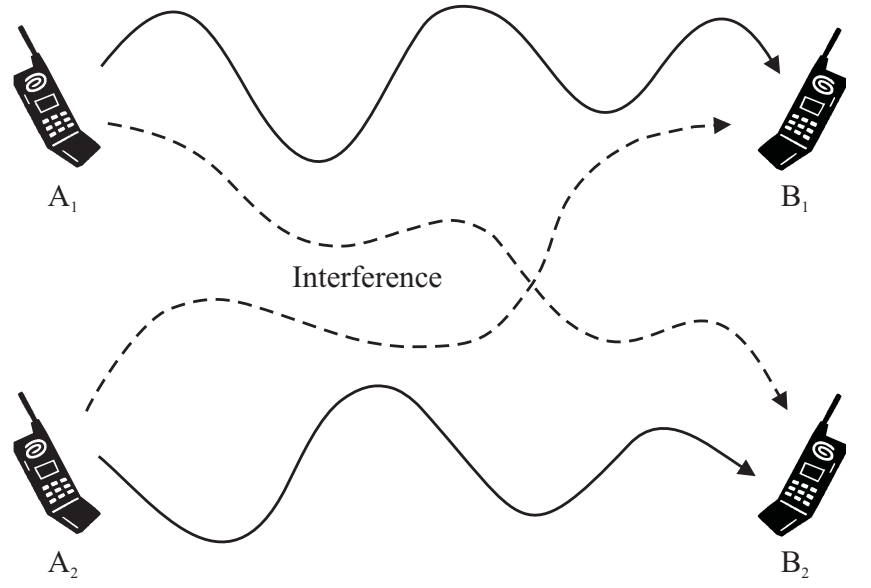

Figure 2: Communication without a master device

which means one is the desired system and the other one is the interferer.

The paper is organized as follows. In Section II two typical interference modes are introduced. Section III describes the system model that was used for simulation. Moreover, definitions on the channel bandwidth, ACI, carrier-to-interference ratio, and guard band are provided. In Section IV the simulation environment is outlined and the results are discussed. Section V concludes the paper and summarizes the results of the new adaptive approaches for efficient spectrum management. Finally, a short outlook on future investigations is highlighted.

\section{INTERFERENCE MODES}

There are two kinds of interference. The first one describes interference between two different users of the same Radio Access Technology (RAT), e.g., two mobiles within a Terrestrial Trunked Radio (TETRA) network. This form of interference is called intra RAT interference. The second one explains interference between two different RATs, e.g., Universal Mobile Telecommunications System (UMTS) and Global System for Mobile Communications (GSM), that is why it is called inter RAT interference. With this background information, it is possible to describe two interference scenarios, namely communication with and without a master device. Fig. 2 and Fig. 3 illustrate these issues.

In Fig. 2 both communication systems must be cognitive radios to be able to observe environmental changes and act accordingly to them. In Fig. 3 both communication systems need not to be cognitive radios, software defined radios are sufficient in this case as no environmental sensing is necessary.

\section{SYSTEM MODEL}

\subsection{General Description}

Consider the model shown in Fig. 4. Let $x_{i}(t)$ denote a MPSK modulated signal, where the index $i, i=1,2, \ldots, N$, represents the $i$-th user, $G_{i}(f)$ is the pulse shaping filter of the $i$-th user $\left(g_{i}(t) \circ \bullet G_{i}(f)\right), f_{c, i}$ is the carrier frequency,
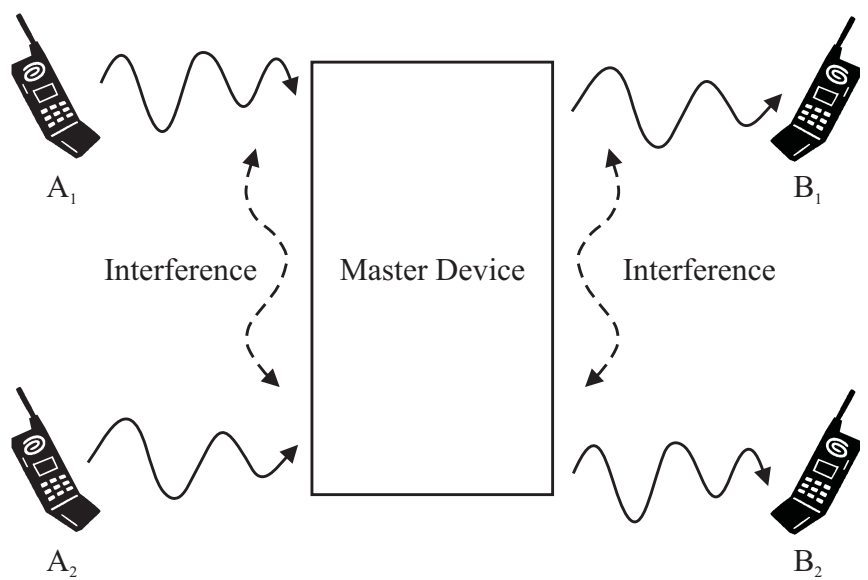

Figure 3: Communication with a master device

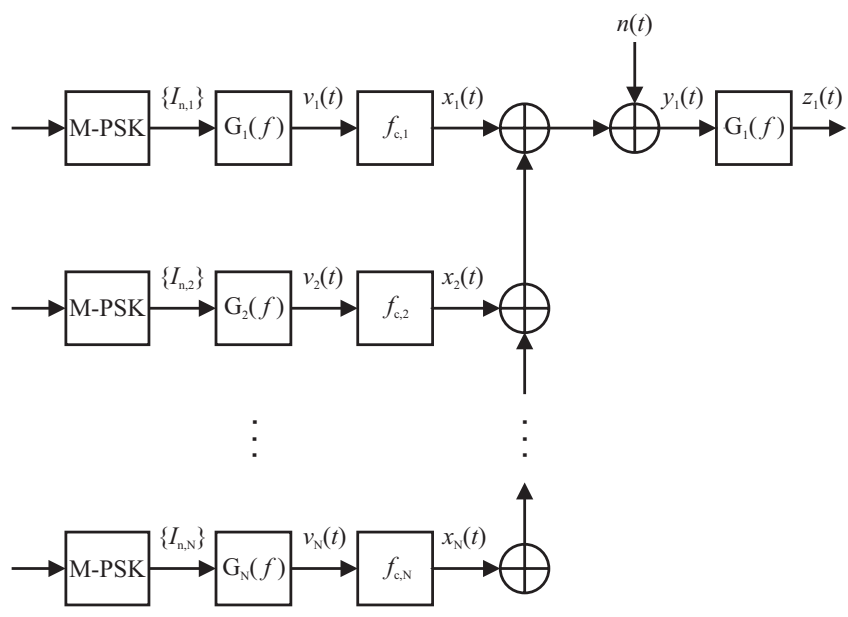

Figure 4: System model

and $\Theta_{i}$ are the random phase angles, respectively.

$$
x_{i}(t)=g_{i}(t) \cdot \cos \left(2 \pi f_{c, i} t+\Theta_{i}\right)
$$

The time variable $t$ lies within the interval $\left[0 ; T_{S}\right]$, where $T_{S}$ is the symbol rate, and the phase angles $\Theta_{i}$ are equally distributed on

$$
\left\{\frac{2 \pi}{M}(m-1), m=1,2, \ldots, M\right\} .
$$

In the simulations only two systems are considered, which means that $i=1,2$, respectively. As pulse shaping filter a Root Raised Cosine (RRC) filter is used. As a consequence, the transmitter and receiver filters are identical. The frequency response of a RRC filter can be expressed as

$$
G_{i}(f)= \begin{cases}1, & |f| \leq \frac{1-\rho_{i}}{2 T} \\ \cos \left[\frac{\pi T}{2 \rho_{i}}\left(|f|-\frac{1-\rho_{i}}{2 T}\right)\right], & \frac{1-\rho_{i}}{2 T} \leq|f| \leq \frac{1+\rho_{i}}{2 T} \\ 0, & |f|>\frac{1+\rho_{i}}{2 T}\end{cases}
$$

The parameter $1 / T$ is the sampling rate of the transmission system and $\rho_{i}$ denotes the roll-off factor $\left(0 \leq \rho_{i} \leq 1\right)$ of the $i$-th user or rather the excess bandwidth [11]. It indicates the extension of bandwidth beyond the Nyquist bandwidth $1 / 2 T$. For example, when $\rho_{i}=0.5$, the excess 
bandwidth is $50 \%$ of the Nyquist bandwidth. According to Fig. 4, the sum of all transmitted signals is influenced by an Additive White Gaussian Noise (AWGN) process, $n(t)$, with the Power Spectral Density (PSD) $\Phi_{n n}(f)=N_{0} / 2$. When two systems are considered, the overall PSD at the receiver filter input is

$$
\Phi_{y_{1} y_{1}}(f)=\Phi_{x_{1} x_{1}}(f)+\Phi_{x_{2} x_{2}}(f)+\frac{N_{0}}{2} .
$$

When $N$ systems with one user, $x_{1}(t)$, and $N-1$ interferers, $x_{i}(t), i=2, \ldots, N$, are considered the overall PSD becomes

$$
\Phi_{y_{1} y_{1}}(f)=\Phi_{x_{1} x_{1}}(f)+\sum_{i=2}^{N} \Phi_{x_{i} x_{i}}(f)+\frac{N_{0}}{2} .
$$

Since M-PSK signals are applied that are shifted by a carrier frequency, $f_{c, i}$, the PSD of a transmit signal can be expressed as

$$
\Phi_{x_{i} x_{i}}(f)=\frac{1}{2}\left[\Phi_{v_{i} v_{i}}\left(f-f_{c, i}\right)+\Phi_{v_{i} v_{i}}\left(-f-f_{c, i}\right)\right],
$$

where $\Phi_{v_{i} v_{i}}(f)$ is the PSD of the equivalent lowpass signal

$$
v_{i}(t)=\sum_{n=-\infty}^{\infty} I_{n, i} \cdot g_{i}\left(t-n T_{S}\right),
$$

and $\left\{I_{n, i}\right\}$ denotes the sequence of symbols that results from the mapping procedure during modulation. After the RRC receiver filter, $G_{1}(f)$, the overall PSD can be described as

$$
\Phi_{z_{1} z_{1}}(f)=\Phi_{y_{1} y_{1}}(f) \cdot\left|G_{1}(f)\right|^{2} .
$$

\subsection{Channel Bandwidth}

Since a RRC filter is used for pulse shaping, it is obvious that the channel bandwidth of the transmission system, $B_{C, i}$, is calculated accordingly to equation (3). Assume the channel bandwidth to correspond to the frequency range in which the frequency response of the filter lies above $70.7 \%$ in relation to full scale (3-dB range). This yields to the following equation:

$$
B_{C, i}=\frac{1}{T}\left(\frac{4 \rho_{i}}{\pi} \arccos \frac{1}{\sqrt[4]{2}}+\left(1-\rho_{i}\right)\right)
$$

As it can be seen, the channel bandwidth depends on the sampling rate of the transmission system, $1 / T$, and the rolloff factor, $\rho_{i}$. For further investigations, we assume the sampling rate to be fixed. However, a first parameter for adopting the channel bandwidth and thus the guard band between two neighboring systems can be identified, the roll-off factor. As a next step, ACI must be considered more precisely in order to find a suitable mathematical formulation for the adaptation algorithm.

\subsection{Adjacent Channel Interference}

A common approach to express ACI is by relating the transmit powers of interfering signals, $x_{i}(t), i=2,3, \ldots, N$, plus noise to the transmit power of the desired signal, $x_{1}(t)$, in a given transmit channel, $B_{C, 1}$, that corresponds to the desired signal. Hence, we get

$$
\mathrm{ACl}=\frac{\int_{B_{C, 1}}\left(\sum_{i=2}^{N} \Phi_{x_{i} x_{i}}(f)+\frac{N_{0}}{2}\right) d f}{\int_{B_{C, 1}} \Phi_{x_{1} x_{1}}(f) d f} .
$$

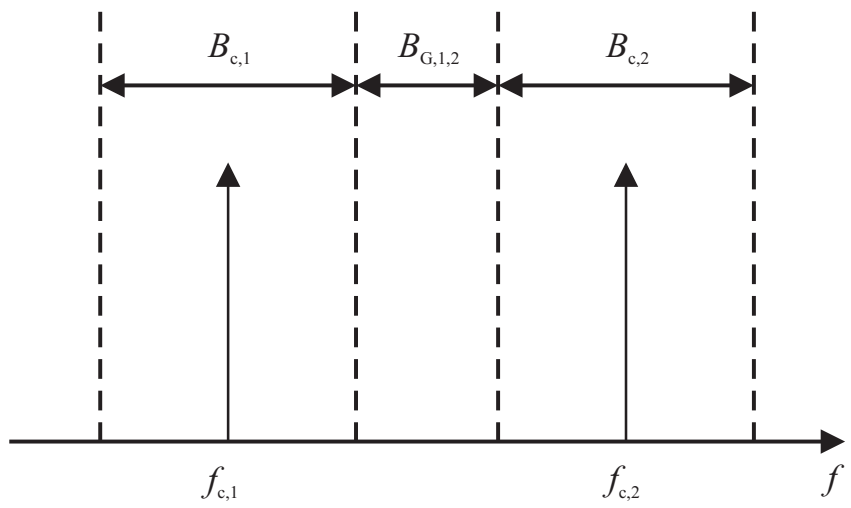

Figure 5: Initial figure for the derivation of the adaptive algorithm

This formula is true, when one desired signal and $N-1$ interferers are considered.

As electrical engineers are used to think in decibel, the carrier-to-interference ratio, $C / I_{A}$, is calculated:

$$
\frac{C}{I_{A}}=10 \cdot \log _{10}\left(\frac{1}{\mathrm{ACl}}\right)
$$

This is an expression that might be compared to the signalto-noise ratio, a common term in wireless communications. Furthermore, it is designated to perform as termination criterion for an adaptive algorithm, i.e., if $C / I_{A}$ is higher than a specific limit, the guard band between consecutive channels can be reduced, thus improving spectral efficiency. The same idea works the other way round. If $C / I_{A}$ is lower than a specific limit, the guard band is widened and, consequently, the quality of service of the transmission is increased.

\subsection{Guard Band}

For the following derivation, we assume the difference $\Delta f$ between two carrier frequencies to be

$$
\Delta f=\left|f_{c, 2}-f_{c, 1}\right| .
$$

This yields the equation (see Fig. 5)

$$
B_{G, 1,2}=\Delta f-\frac{1}{2}\left(B_{C, 1}+B_{C, 2}\right) .
$$

If $f_{c, 2} \geq f_{c, 1}, B_{G, 1,2}$ can be expressed as follows (see (9)):

$$
\begin{aligned}
B_{G, 1,2}= & f_{c, 2}-f_{c, 1} \\
& -\frac{2}{\pi T} \arccos \frac{1}{\sqrt[4]{2}} \cdot\left(\rho_{1}+\rho_{2}\right) \\
& +\frac{1}{2 T}\left(2-\rho_{1}-\rho_{2}\right)
\end{aligned}
$$

Supposing that both systems transmit with equal power, four parameters in order to adapt the guard band dynamically are gained. These are:

$$
\left\{\rho_{i}\right\}_{i=1}^{2},\left\{f_{c, i}\right\}_{i=1}^{2}
$$

In a first consideration, let the roll-off factors be equal and constant:

$$
\rho_{1}=\rho_{2}=\rho
$$



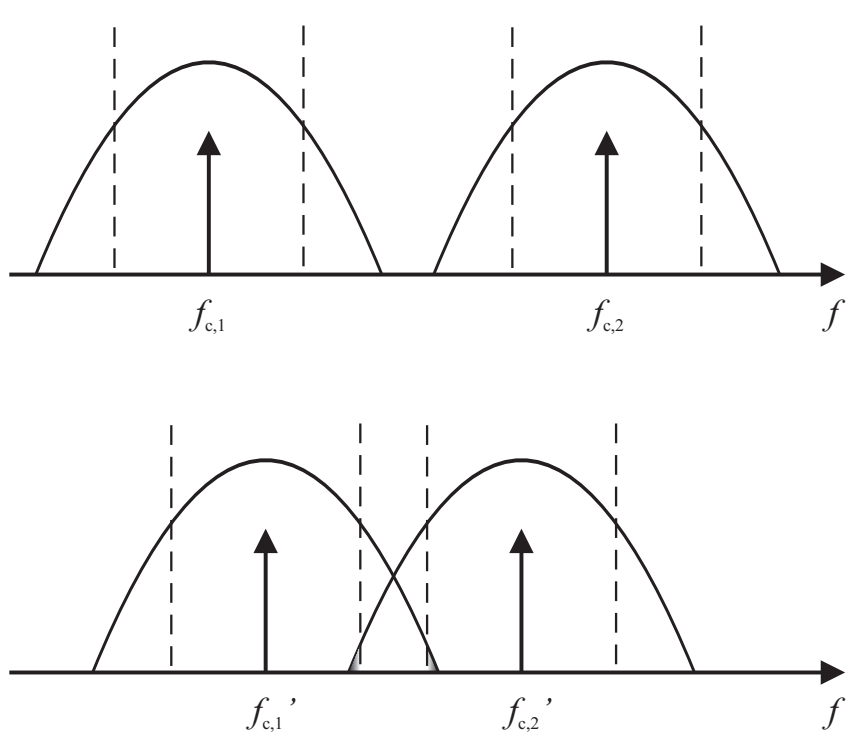

Figure 6: Shifting the carrier frequencies

Thus, the guard band results in the equation

$$
B_{G, 1,2}=f_{c, 2}-f_{c, 1}-B_{C}
$$

where $B_{C}$ is a constant value. In a second consideration, let the carrier frequencies be fixed. As a consequence, $\Delta f$ is constant:

$$
f_{c, 1}=\text { const, } f_{c, 2}=\text { const } \rightarrow \Delta f=\text { const }
$$

Herefrom, a mathematical description of the guard band results that depends on both $\rho_{1}$ and $\rho_{2}$ as well:

$$
\begin{aligned}
B_{G, 1,2}= & \Delta f-\frac{2}{\pi T} \arccos \frac{1}{\sqrt[4]{2}} \cdot\left(\rho_{1}+\rho_{2}\right) \\
& +\frac{1}{2 T}\left(2-\rho_{1}-\rho_{2}\right)
\end{aligned}
$$

Now, an adaptive algorithm can be formulated that is able to control the guard band dynamically according to transmission environment. As termination criterion a value of $C / I_{A_{\text {min }}}=20 \mathrm{~dB}$ is used here.

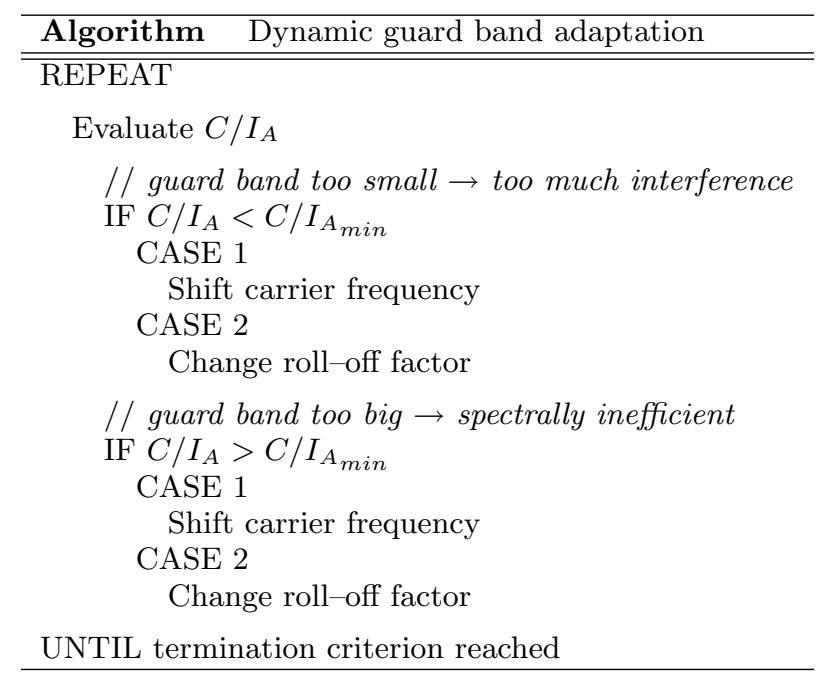

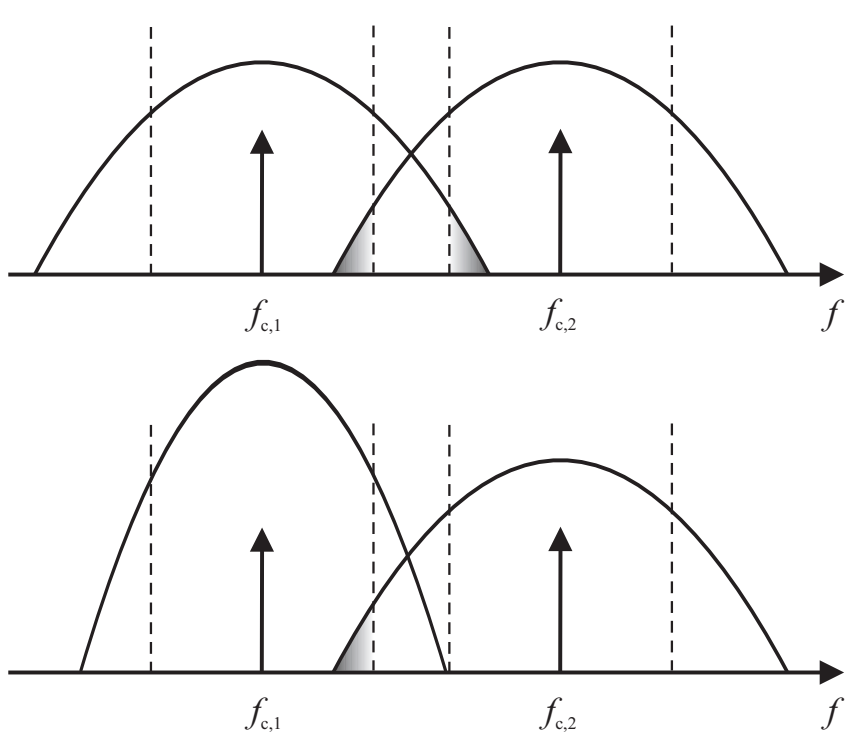

Figure 7: Changing the roll-off factor

Nevertheless, one point remains open: How does a receiver know which part of the received signal belongs to the desired signal and which part to the interferer? A first and more obvious approach is to communicate with a master device, let's say, e.g., a base station in mobile communication systems. A second one might be the use of cognitive radios that are able to detect desired and undesired signals by blind source separation ${ }^{1}$.

\section{SIMULATION}

\subsection{Simulation Environment}

In simulations a gray-coded 4-PSK signal with an initial phase of $\pi / 4$ is used. Two signals are provided, one desired signal and one interferer. The channel bandwidth is calculated dynamically (see equation (9)). The system behavior with respect to several carrier frequency spacings - which means different guard band widths - and different roll-off factors is considered. First, $\mathrm{ACl}$ and $C / I_{A}$ have been investigated. In addition, a bit error rate, BER, graph is provided in which the simulation results are compared to the theoretical boundary of a 4-PSK modulated signal transmitted through an AWGN-channel.

\subsection{Simulation Results}

In Fig. 8 the behavior of adjacent channel interference, $\mathrm{ACl}$, in relation to the normalized carrier frequency spacing, $\Delta f / f_{s}$, can be seen, where $f_{s}$ denotes the sampling frequency of our simulation system. As expected, $\mathrm{ACl}$ decreases if the difference between the two carrier frequencies grows. Especially at $\Delta f / f_{s}=1$ there is hardly any $\mathrm{ACl}$. Fig. 9 shows the progression of the carrier-to-interference ratio, $C / I_{A}$. Of course, it increases as the carrier frequency spacing grows. These graphs are calculated with respect to equation (11). In Fig. $10 C / I_{A}$ is shown again, but this time in relation to

\footnotetext{
${ }^{1}$ Blind Source Separation (BSS) aims at recovering single signals from a mixture of observed signals by exploiting the assumption of mutual statistical independence between the signals. A famous problem that deals with BSS is the "cocktail party problem."
} 


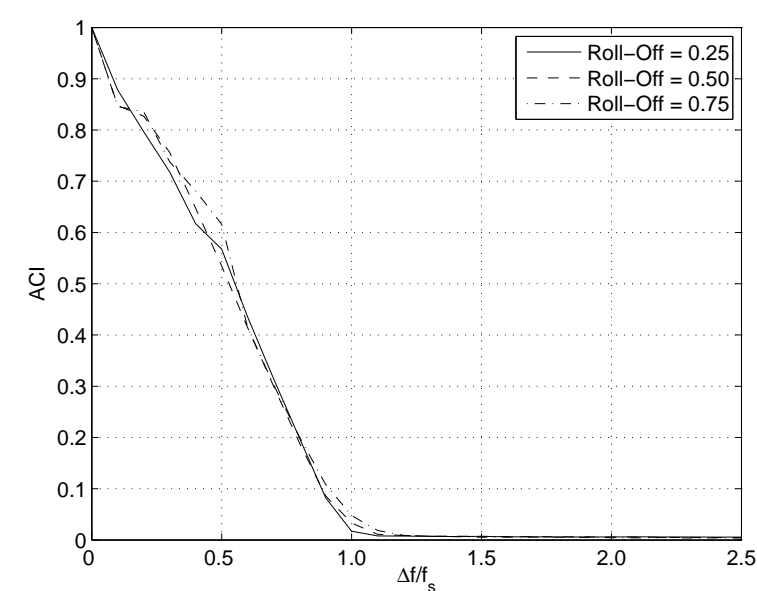

Figure 8: Adjacent channel interference at $E_{b} / N_{0}=$ $20 \mathrm{~dB}$

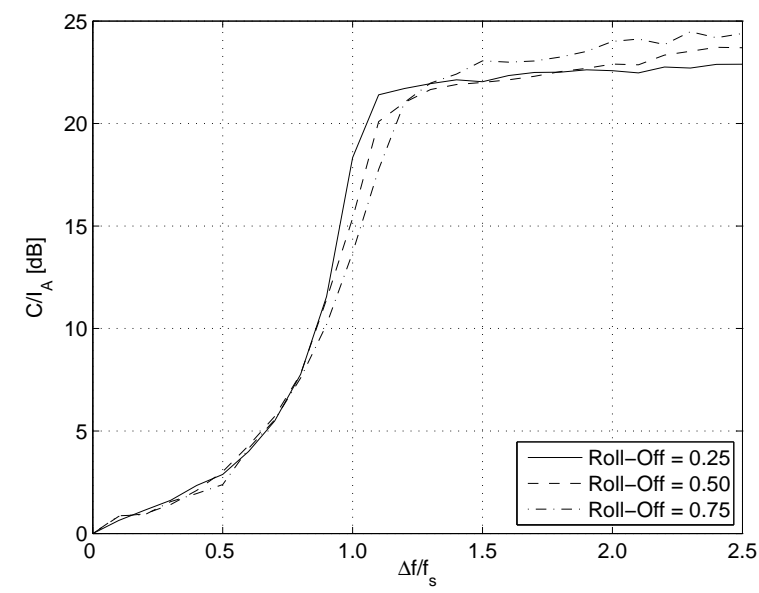

Figure 9: Carrier-to-interference ratio at $E_{b} / N_{0}=$ $20 \mathrm{~dB}$

the roll-off factor which is equal for both systems in this case. It can be seen, that there is hardly any influence on $C / I_{A}$, if the normalized difference between the carrier frequencies is either smaller or larger than 1.0. Hence, it makes no sense to alter the roll-off factor in those cases. Finally, Fig. 11 represents the bit error rate of system 1 when it is disturbed by system 2 . It can be seen, that voice traffic $\left(\mathrm{BER} \approx 10^{-3}\right)$ is possible if only 1 decibel of $E_{b} / N_{0}$ is invested additionally, however, with the benefit of saved overall bandwidth.

\section{CONCLUSION AND FUTURE WORK}

New resource allocation techniques must be developed in order to match the growing demand for spectrum. In this paper the influence of ACI between two systems with respect to the difference between the two carrier frequencies on the one hand and the roll-off factors on the other hand has been investigated. It has been shown by simulation, that it is possible to increase spectral efficiency by either shifting

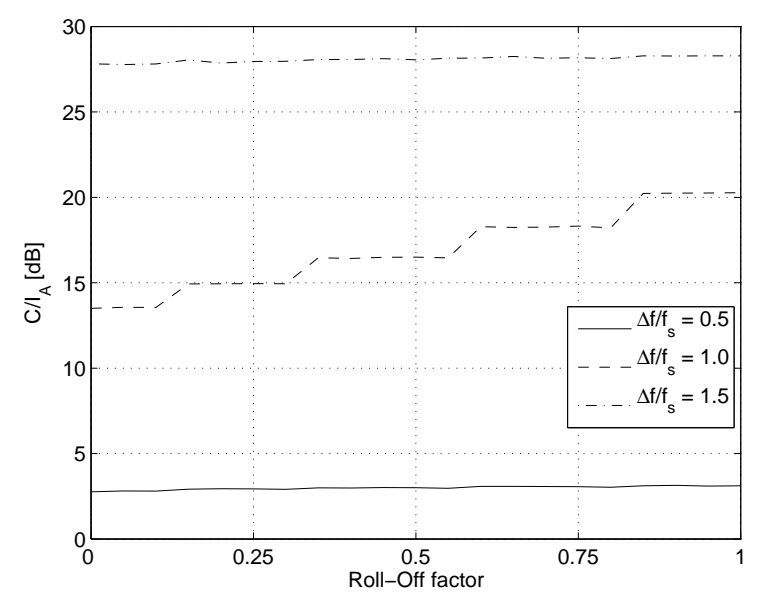

Figure 10: Carrier-to-interference ratio versus rolloff factor $\rho$

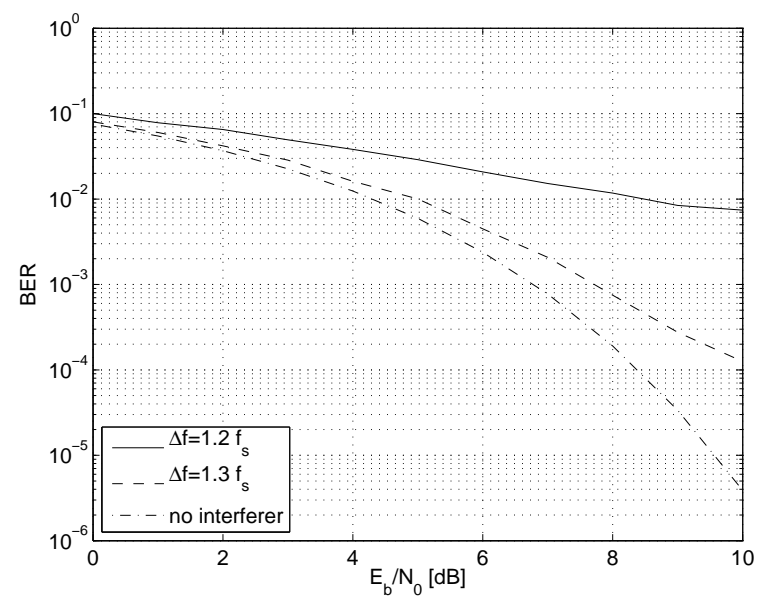

Figure 11: Bit error rate (BER) with the roll-off factors $\rho_{1}=0.25$ and $\rho_{2}=0.50$, respectively

the carrier frequencies closer together or altering the roll-off factors. Nevertheless, the latter approach does not seem to be as promising as the first. A first concept for an adaptive algorithm has been presented that is able to control the guard band dynamically.

A lot of points are still open and, hence, a lot of future work must be done. The simulation model must be widened to several users. In addition, communication with or without a master device must be taken under closer consideration. A very interesting aspect is the creation of a kind of avalanche effect that might arise during shifting. Of course, this can lead to disastrous impacts and must be avoided. Another open question is who is actually shifting its frequency? A fair distribution among the users in a network must be guaranteed. Furthermore, one is faced with the question of how the shifting will be done? In discrete steps until the termination criterion is reached? This might lead to the effect that the users overlap for a short time which might decrease 
the quality of service. Who will participate in the spectrum sharing process - everyone or just a few selected users? And if only a small selection will participate, how is this selection done?

These issues are currently under investigation and this dynamic spectrum sharing algorithm is expected to be very promising.

\section{Acknowledgment}

This work has been performed in the framework of the EU funded project End-to-End Reconfigurability $\left(\mathrm{E}^{2} \mathrm{R}\right)$. The authors would like to acknowledge the contributions of their colleagues from $\mathrm{E}^{2} \mathrm{R}$ consortium.

\section{REFERENCES}

[1] J. Dumont, S. Lasaulce, and JM. Chaufray. Adjacent channel interference in WCDMA networks equipped with multiple antennas mobile stations. In Signal Processing for Advanced Wireless Communications (SPAWC), July 2004

[2] D. E. Thompson. Modelling adjacent channel interference in 3G networks. In 5th European Personal Mobile Communications Conference (EMPCC 2003), Glasgow, Scotland, April 2003.

[3] B. R. Petersen and D. D. Falconer. Suppression of adjacent-channel, cochannel, and intersymbol interference by equalizers and linear combiners. In IEEE Trans. Commun., volume 42, pages 3109-3118, December 1994.

[4] H. Arslan, S. C. Gupta, G. E. Bottomley, and S. Chennakeshu. Successive cancellation of adjacent channel signals in FDMA/TDMA digital mobile radio systems. In Proc. IEEE Veh. Technol. Conf. (VTC '98), pages 1720-1724, Ottawa, Ontario, Canada, May 1998.
[5] H. Arslan, S. C. Gupta, G. E. Bottomley, and S. Chennakeshu. Joint demodulation for the suppression of ACI in FDMA/TDMA mobile radio systems. In Proc. IEEE Intl. Conf. Commun. (ICC '98), pages 723-724, Atlanta, GA, June 1998.

[6] A. Schlereth. Digital algorithms for suppression of adjacent channel interference in FM-receivers. In IEEE Intl. Conf. on Acoustics, Speech, and Signal Processing (ICASSP '93), volume 3, pages 5-8, Minneapolis, MN, 1993.

[7] S. Golestaneh, H. M. Hafez, and S. A. Mahmoud. The effects of adjacent channel interference on the capacity of FDMA cellular systems. In IEEE Trans. Veh. Technol., volume 43, pages 946-954, 1994.

[8] P. T. Mathiopoulos, J. S. Toor, and G. K. Karagiannidis. Effects of ACI and nonlinearities on the performance of differentially detected GMSK signals. In IEE Proc.-Commun., volume 151, 2004.

[9] B. Vaghani and C. Beard. Improving spectral efficiency and reducing adjacent channel interference of a wireless emergency communication system.

[10] B. Vaghani and C. Beard. A scheme to measure error rates in the presence of adjacent channel interference using frequency domain analysis. In IASTED International Conference on Communications, Internet, and Information Technology (CIIT 2004), St. Thomas, US Virgin Islands, November 2004.

[11] J. G. Proakis. Digital Communications. McGraw-Hill, New York, 4th edition, 2001. 